\title{
Diversidade de fungos micorrízicos arbusculares em área de Caatinga, $\mathrm{PE}$, Brasil $^{1}$
}

\author{
Catarina Maria Aragão de Mello ${ }^{2,4}$, Iolanda Ramalho da Silva², Juliana Souza de Pontes², \\ Bruno Tomio Goto ${ }^{3}$, Gladstone Alves da Silva ${ }^{2}$ e Leonor Costa Maia ${ }^{2}$
}

Recebido em 18/08/2011. Aceito em 20/07/2012

\section{RESUMO}

(Diversidade de fungos micorrízicos arbusculares em área de Caatinga, PE, Brasil). O objetivo deste trabalho foi o de determinar a infectividade, a riqueza de FMA e a condição micorrízica da vegetação em área de Caatinga não antropizada. Coleta de solo e raízes foi realizada em Triunfo/PE. Raízes foram examinadas para determinação da colonização micorrízica. O número mais provável (NMP) de propágulos infectivos de FMA foi estimado por diluições sucessivas do solo e a utilização de milho (Zea mays L.) como planta isca. Glomerosporos foram quantificados e preparados em lâminas, para identificação das espécies. As plantas apresentaram-se pouco colonizadas (média < 10\%) e o número de glomerosporos foi baixo ( $<1$ glomerosporo $\mathrm{g}^{-1} \mathrm{de}$ solo), como registrado em outras áreas de Caatinga.

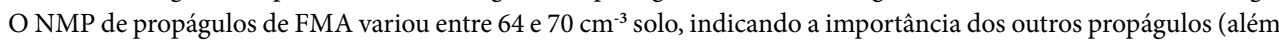
dos glomerosporos) na dispersão dos FMA. Foram registrados 16 táxons de FMA, dos gêneros Acaulospora, Entrophospora, Gigaspora, Glomus, Kuklospora, Pacispora e Paraglomus; Glomus foi o mais representativo (sete espécies). Pacispora boliviana está sendo relatada pela primeira vez no Brasil. Os elevados teores de P na área provavelmente contribuiram para os baixos valores de colonização, infectividade e riqueza de FMA na área estudada. Confirma-se que os FMA são comuns em solos do semiárido brasileiro, com espécies de Glomus predominando nesse ambiente.

Palavra-Chave: Glomeromycota, Ocorrência, Semiárido

\begin{abstract}
(Diversity of arbuscular mycorrizal fungi in an area of Caatinga, PE, Brazil). The objective of this work was to determine the infectivity and richness of AMF and the mycorrhizal conditions of the vegetation in a preserved area of Caatinga. Soil and root samples were taken in two subareas in the municipality of Triunfo, PE. The roots were stained with Trypan blue, and observed with a microscope to determine if colonization of mycorrhizal fungi was present. Glomerospores were extracted from soil, counted, mounted on microscope slides and the AMF species were identified. The plants from the area showed $<10 \%$ colonization. The number of glomerospores $\left(<1 \mathrm{~g}^{-1}\right.$ soil $)$ did not differ between the subareas although in one area the amount of $\mathrm{P}$ in the soil was three times higher than in the other. The number of AMF propagules varied from 64 to $70 \mathrm{~cm}^{-3}$ in the soil. Sixteen taxa of AMF were registered and Glomus was the most representative genus, with seven species. Pacispora boliviana is reported for the first time in Brazil. The high amount of $\mathrm{P}$ probably contributes to the low values of propagules, colonization, infectivity and richness of AMF in the area. AMF have been commonly found in the Brazilian semiarid region and species of Glomus are predominant in this environment.
\end{abstract}

Key words: Glomeromycota, Occurrence, Semi-arid

\footnotetext{
${ }^{1}$ Parte da dissertação de Mestrado da primeira Autora

${ }^{2}$ Universidade Federal de Pernambuco, Departamento de Micologia, Laboratório de Micorrizas, Recife, PE, Brasil

${ }^{3}$ Universidade Federal do Rio Grande do Norte, Programa de Pós-Graduação em Sistemática e Evolução, Departamento de Botânica Ecologia e Zoologia, Natal, RN, Brasil

${ }^{4}$ Autor para correspondência: meloearagao@yahoo.com.br
} 


\section{Introdução}

Florestas tropicais e subtropicais ocupam cerca de $40 \%$ da crosta terrestre e destes as florestas secas, que incluem a Caatinga, abrangem $42 \%$ (Moreira et al. 2006). A Caatinga compreende aproximadamente $70 \%$ da região Nordeste e parte do norte de Minas Gerais, representando cerca de 11\% do território nacional. O clima característico é semiárido, com altas temperaturas, precipitações escassas e irregulares, concentradas em dois a cinco meses (janeiro a maio) ao ano. A região também apresenta características meteorológicas extremas, tais como os mais altos níveis de radiação solar e temperatura média anual $\left(26-28^{\circ} \mathrm{C}\right)$ do país, além de baixa taxa de umidade relativa (50\%) e baixa nebulosidade, e elevada evaporação (2000 $\mathrm{mm} \mathrm{ano}^{-1}$ ) (Drumond et al. 2000; Moura et al. 2007).

Apesar das condições extremas, estudos comprovam que a Caatinga é rica em espécies animais e vegetais, muitas das quais endêmicas, contradizendo a teoria de que esse bioma era improdutivo e pobre em diversidade (Leal et al. 2003). A Caatinga encontra-se bastante alterada pela perturbação e degradação ambientais causadas pelo uso irracional dos recursos naturais. Isso incluiu atividades agrícolas, queimadas, extrativismo mineral e vegetal e pecuária extensiva, o que tem levado à rápida perda de espécies endêmicas e de processos ecológicos importantes para manutenção dos seus ecossistemas (Araújo et al. 2005).

Os fungos micorrízicos arbusculares (FMA), filo Glomeromycota (Schüssler et al. 2001), formam a mais ampla simbiose entre fungos e plantas na natureza e podem ser encontrados nos mais variados biomas e ecossistemas, tais como florestas tropicais, desertos, savanas, pradarias e dunas, ocupando áreas naturais ou agrícolas, preservadas ou degradadas (Smith \& Read 1997). Os FMA promovem maior absorção de nutrientes, beneficiando o hospedeiro principalmente com a translocação do fósforo (He et al. 2002), e também outros íons, proporcionam aumento da resistência vegetal contra patógenos (Liu et al. 2007) e maior tolerância a estresses hídrico (Beltrano \& Ronco 2008) e salino (Maia \& Yano-Melo 2005), contribuindo ainda para a agregação do solo (Mergulhão et al. 2010). Além disso, atuam na definição de nichos ecológicos ocupados pelos vegetais, determinando a composição das comunidades de plantas (Francis \& Read 1995). Considerando que os FMA participam de processos importantes para a manutenção dos ambientes terrestres, estudos sobre esses fungos podem contribuir para esclarecer aspectos importantes da sua atuação e funcionalidade na Caatinga. Nesse contexto, foi determinada a condição micorrízica das plantas, a infectividade e a riqueza de FMA em uma área de Caatinga preservada, no estado de Pernambuco.

\section{Material e métodos}

Coleta de solo e raízes foi realizada em outubro/2008 (estação seca) em Triunfo ( $7^{\circ} 50^{\prime} 17^{\prime \prime}$ S e $38^{\circ} 06^{\prime} 06^{\prime \prime} \mathrm{W}$ ), no sertão de Pernambuco. Esse município está inserido na bacia hidrográfica do rio Pajeú, com relevo forte-ondulado e montanhoso; os solos são do tipo cambissolos latossólicos, com vegetação predominante do tipo floresta subcaducifólia; a pluviometria anual chega a $1220 \mathrm{~mm}$ (CPRM 2005) e a temperatura média anual varia de 16 a $26^{\circ} \mathrm{C}$. O local de coleta foi o sítio "Carro quebrado" (70 $52^{\prime} 28^{\circ}$ " S e $38^{\circ}$ 06 ” 03 ” W), apresenta vegetação primária distribuída em aproximadamente 880 hectares e $650 \mathrm{~m}$ de altitude. É uma área de Caatinga bem preservada sem pressão antrópica.

$\mathrm{Na}$ área predominam espécies das famílias: Anacardiaceae (Myracrodruon urundeuva Engl.), Apocynaceae (Aspidosperma pyrifolium Mart.), Bignoniaceae (Tabebuia impetiginosa (Mart. Ex DC.) Standl., Boraginaceae (Cordia leucocephalla Moricand.), Bromeliaceae (Bromelia sp.), Euphorbiaceae (Manihot pseudoglaziovii Pax. et K. Hoffman), Fabaceae [Mimosa tenuiflora (Willd.) Poiret] e Rhamnaceae (Zizyphus joazeiro Mart.), como referido também em área próxima, na mesma altitude (Ferraz et al. 2003). As plantas são de grande porte, possivelmente devido às boas condições hídricas locais.

Para coleta, foram delimitadas duas subáreas $(10$ x 100 $\mathrm{m})$, distantes entre si 10 metros, e retirou-se, em cada uma (0-20 cm de profundidade), 10 amostras de solo rizosférico (compostas de 3 subamostras), coletadas aleatoriamente. Parte do solo foi enviada à Estação Experimental de Cana-de-açúcar do Carpina, pertencente à Universidade Federal Rural de Pernambuco (UFRPE) para análises químicas e ao Instituto Agronômico de Pernambuco - IPA, para análises físicas (Tab. 1); o restante do solo foi usado para as demais análises, relativas aos FMA.

O Número Mais Provável (NMP) de propágulos infectivos foi estimado de acordo com Feldmann \& Idczak (1992). Parte das dez amostras de solo foi misturada, homogeneizada, seca e peneirada (malha de $0,5 \mathrm{~cm}$ de abertura), constituindo a amostra base para as diluições (solo-inóculo). $\mathrm{O}$ solo diluente, proveniente do local de coleta, foi igualmente homogeneizado e peneirado, sendo depois autoclavado por $1 \mathrm{~h}$ a $120^{\circ} \mathrm{C}$, durante quatro dias consecutivos, seco em estufa a $105^{\circ} \mathrm{C}$ e usado após 20 dias. Foram realizadas diluições sucessivas do solo-inóculo em recipientes de 200 $\mathrm{mL}$, além do solo não diluído (0); para a primeira diluição (1:10), $20 \mathrm{~mL}$ do solo-inóculo foi misturado a $180 \mathrm{~mL}$ do diluente. As diluições subseqüentes foram determinadas nas proporções de 1:100 e 1:1000 a partir do solo-inóculo. Para cada diluição foram usadas cinco repetições, totalizando 20 potes por subárea amostrada. Como planta isca foi usado milho (Zea mays L.). Para a determinação do NMP as plântulas foram colhidas após um mês, as raízes foram lavadas, diafanizadas e coradas com azul de tripano (Phillips \& Hayman 1970) e observadas ao microscópio para verificação da presença de associação micorrízica. Foi considerada colonizada a planta que apresentou uma das seguintes estruturas próprias de FMA: arbúsculos, vesículas e/ou esporos e hifas. O NMP de propágulos infectivos de 
Tabela 1. Análise química e granulométrica do solo em duas subáreas (A1 e A2) de Caatinga em Triunfo - PE.

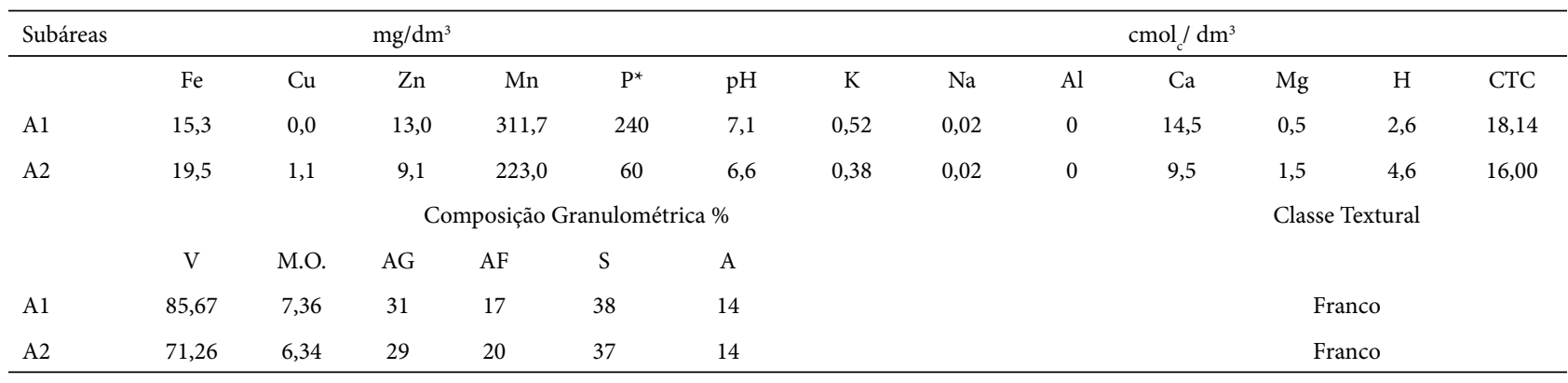

$\mathrm{CTC}=$ capacidade de troca de cátions; $\mathrm{V}=$ saturação por bases (\%); M.O. = matéria orgânica (\%); $\mathrm{AG}=$ areia grossa; $\mathrm{AF}=$ areia fina; $\mathrm{S}=$ silte; $\mathrm{A}=$ argila.${ }^{*}$ fósforo disponível pelo método extrator de Mehlich 1.

FMA $\mathrm{g}^{-1}$ de solo foi obtido comparando-se os resultados com os dados da tabela de Cochran (1950).

As raízes provenientes do campo foram preparadas para análise como mencionado (Phillips \& Hayman 1970); as muito pigmentadas foram clareadas em $\mathrm{H}_{2} \mathrm{O}_{2}$, submetidas a aquecimento a $90^{\circ} \mathrm{C}$ durante $10 \mathrm{~min}$. Cem fragmentos de raízes, de cada ponto de coleta, foram montados entre lâmina e lamínula e examinados ao microscópio, para cálculo da colonização radicular (Giovanetti \& Mosse 1980).

Glomerosporos e esporocarpos de FMA foram extraídos de $20 \mathrm{~g}$ de solo seco, pela técnica da decantação e peneiramento úmido (Gerdemann \& Nicolson 1964), seguido por centrifugação em sacarose (Jenkins 1964) e quantificados em placas canaletadas sob estereomicroscópio ( $40 \mathrm{x}$ ). Cada esporocarpo foi contado como uma unidade de propágulo. Os glomerosporos foram montados em lâminas com polivinil lacto-glicerol (PVLG) e PVLG + reagente de Melzer, examinados em microscópio e as espécies identificadas após consulta ao manual de identificação de FMA (Schenck \& Pérez 1990), e sites específicos (http://invam.caf.wvu.edu; http://www.agro.ar.szczecin.pl/ jblaszkowski/).

Os dados de colonização e de número de esporos foram transformados em arco seno e $(\log \mathrm{x}+1)$, respectivamente e submetidos à análise de variância (ANOVA). As médias foram comparadas pelo teste de Tukey $(\mathrm{P} \leq 1 \%)$ usando o programa Statistica (Statsoft 1995).

\section{Resultados e discussão}

A média de colonização radicular das plantas foi inferior a $10 \%$ (Tab. 2), o que pode ser considerado baixo em comparação com outra área de Caatinga, onde se registrou $56,8 \%$, em Pernambuco (Mergulhão et al. 2007) e de 5 a 80 $\%$, em áreas de Alagoas (Souza et al. 2003). A quantidade de nutrientes disponíveis para a planta afeta a abundância e a colonização por FMA (He et al. 2002; Panwar \& Tarafdar 2006; Mathur et al. 2007). Baixas ou altas concentrações de fósforo no solo podem aumentar ou suprimir a penetração das hifas nas raízes e, consequentemente, a colonização micorrízica (Aguilera et al. 1998). Esse talvez tenha sido o
Tabela 2. Colonização micorrízica, número de glomerosporos e número mais provável (NMP) de propágulos infectivos de FMA em duas subáreas (A1 e A2) de Caatinga em Triunfo, PE.

\begin{tabular}{lccc}
\hline Áreas & Colonização $(\%)$ & $\begin{array}{c}\mathrm{N}^{\circ} \text { de } \\
\text { glomerosporos } \\
\left(\mathrm{g}^{-1} \text { solo }\right)\end{array}$ & $\begin{array}{c}\mathrm{NMP} \\
\left(\mathrm{cm}^{-3} \text { solo }\right)\end{array}$ \\
\hline $\mathrm{A} 1$ & $8,2 \mathrm{a}$ & $0,20 \mathrm{a}$ & 70 \\
$\mathrm{~A} 2$ & $10,0 \mathrm{a}$ & $0,24 \mathrm{a}$ & 64 \\
\hline
\end{tabular}

Médias seguidas pela mesma letra, na coluna, não diferem significativamente pelo teste de Tukey a $1 \%$.

caso, considerando os elevados valores de fósforo nos solos analisados, indicando que neste local há um veio desse elemento (E. Sampaio, comunicação pessoal). A planta e o fungo são influenciados por suas próprias necessidades e as do parceiro e as condições do ambiente sujeitam a simbiose a mudanças (Clark et al. 2009).

O número de glomerosporos ( $1 \mathrm{~g}^{-1}$ solo) também foi baixo (Tab. 2). Vários trabalhos relatam o baixo número de esporos de FMA em áreas preservadas do semiárido brasileiro (Carvalho \& Alencar 2000; Souza et al. 2003; Borba \& Amorim 2007). Foram encontrados 64 e 70 propágulos de FMA $\mathrm{cm}^{-3}$ solo, número muito superior ao de glomerosporos, o que demonstra que outros tipos de estruturas micorrízicas são importantes para propagação dos FMA em solos da Caatinga. Em outras áreas de Caatinga preservadas nos estados de Alagoas e Pernambuco, o número de propágulos de FMA variou de $13 \mathrm{~g}^{-1}$ solo (Souza et al. 2003) a $130 \mathrm{~cm}^{-3}$ solo (Mergulhão et al. 2007), respectivamente. Por outro lado, estudos em áreas impactadas por mineração, em comparação com áreas próximas, preservadas, indicaram variação de 0 a 36 propágulos de FMA g ${ }^{-1}$ de solo (arredores de mineração de cobre) (Silva et al. 2001), e de 17 a 540 propágulos $\mathrm{cm}^{-3}$ de solo (arredores de mineração de gipsita) (Mergulhão et al. 2007), respectivamente. Essa variação no número de propágulos de FMA reflete diferenças não apenas na comunidade vegetal e de fungos, mas, principalmente, em relação à composição química e uso do solo.

Como observado neste estudo (Tab. 1) e outros trabalhos, há diminuição da produção de glomerosporos (Douds 
1994; Souza et al. 2003) e demais propágulos micorrízicos (Covacevich et al. 2006; Karanika et al. 2008), assim como da colonização em solos com níveis elevados de $\mathrm{P}$ no solo. $\mathrm{O}$ alto teor de matéria orgânica encontrado na área também pode ter favorecido o desenvolvimento de organismos predadores de glomerosporos, contribuindo para o baixo número de esporos. O nível de colonização também é influenciado pela demanda nutricional das plantas hospedeiras (Muthukumar \& Udaiyan 2002) e, uma vez que existam nutrientes disponíveis no solo, não há necessidade de formação da simbiose, a menos que a planta seja micorrízica obrigatória.

Foram registrados 16 táxons de FMA (Tab. 3) e Glomus foi o gênero mais representativo, com sete espécies. Alguns representantes desse gênero não puderam ser identificados ao nível específico, pois os esporos não estavam em condições adequadas para estudo. Não se encontrou táxons escutelosporóides; a ausência desses táxons pode ser resultado de pressão de seleção (Singh et al. 2008), considerando o ambiente peculiar da Caatinga. Em certos casos, representantes desses táxons são identificados apenas após alguns ciclos de culturas armadilha (Yano-Melo et al.2003) o que indica que estão na rizosfera sob a forma de micélio não sendo, portanto, detectados. Em culturas armadilha as condições podem se tornar mais propícias para esporulação dos FMA, permitindo a identificação das espécies. Representantes de Glomus e algumas espécies de Acaulospora passam por período de dormência e geralmente produzem esporos pequenos e de crescimento rápido, os quais são mais facilmente propagados e têm mais possibilidade de sobrevivência (Shi et al. 2007; Zhao \& Zhao 2007). Entre as espécies de FMA encontradas, Pacispora boliviana é relatada pela primeira vez para o Brasil, uma vez, que até o momento este táxon era de ocorrência apenas na Bolívia (Oehl \& Sieverding 2004), enquanto as demais espécies são comuns a áreas de Caatinga (Goto et al. 2010).

A riqueza de espécies (16) foi menor do que tem sido mencionado para outras áreas de Caatinga preservada, nos estados de Alagoas e Pernambuco (Souza et al. 2003; Silva et al. 2007; ), mas supera dados referentes a regiões áridas da Namíbia e da Jordânia, onde apenas 12 e oito espécies de FMA foram identificadas, respectivamente (Uhlmann et al. 2006; Mohammad et al. 2003). O alto nível de fósforo nas áreas estudadas pode ter influenciado negativamente a ocorrência dos FMA, prejudicando a colonização e a produção de glomerosporos. Em geral, solos com baixo nível de $\mathrm{P}\left(<40 \mathrm{mg} \mathrm{dm}^{-3}\right)$ são mais ricos em espécies de FMA (Souza et al. 2003; Collins \& Foster 2009) e naqueles com concentração de fósforo $>40 \mathrm{mg} \mathrm{dm}^{-3}$ a esporulação e a diversidade de FMA são negativamente afetados (Souza et al. 2003; Hijri et al. 2006; Gai et al. 2009).

Embora a riqueza de espécies tenha sido similar entre as subáreas ( 9 e 10 espécies, respectivamente), apenas três táxons foram comuns a ambas: G. sinuosum, Glomus sp. 5 e Kuklospora colombiana, as quais, assim como as demais espécies, foram referidas em outras áreas de Caatinga (Mergulhão et al. 2010; Silva et al. 2005).

Os FMA são componentes comuns da microbiota edáfica na região semiárida brasileira, com espécies de Glomus predominando neste ambiente; entretanto, em algumas

Tabela 3. Espécies de fungos micorrízicos arbusculares em área de Caatinga em Triunfo, PE.

\begin{tabular}{|c|c|c|}
\hline \multirow{2}{*}{ Espécies } & \multicolumn{2}{|c|}{ Subáreas } \\
\hline & A1 & A2 \\
\hline Acaulospora morrowiae Spain \& N.C. Schenck & & $\mathrm{X}$ \\
\hline A. scrobiculata Trappe & & $\mathrm{X}$ \\
\hline A. spinosa C. Walker \& Trappe & $\mathrm{X}$ & \\
\hline Entrophospora infrequens (I.R. Hall) R.N. Ames \& R.W. Schneid. & $\mathrm{X}$ & \\
\hline Gigaspora decipiens I.R. Hall \& L.K. Abbott & & $\mathrm{X}$ \\
\hline Gigaspora sp. & & $\mathrm{X}$ \\
\hline Glomus coremioides (Berk. \& Broome) D. Redecker \& J.B. Morton & $\mathrm{x}$ & \\
\hline G. sinuosum R.T. Almeida \& N.C. Schenck & $\mathrm{X}$ & $\mathrm{X}$ \\
\hline Glomus sp.1 & $\mathrm{X}$ & \\
\hline Glomus sp.2 & & $\mathrm{X}$ \\
\hline Glomus sp.3 & $\mathrm{X}$ & \\
\hline Glomus sp.4 & $\mathrm{X}$ & \\
\hline Glomus sp.5 & $\mathrm{X}$ & $\mathrm{X}$ \\
\hline Kuklospora colombiana (Spain \& N.C. Schenck) Oehl \& Sieverd. & $\mathrm{X}$ & $\mathrm{X}$ \\
\hline Pacispora boliviana Sieverd. \& Oehl & & $\mathrm{X}$ \\
\hline Paraglomus occultum (C. Walker) J.B. Morton \& D. Redecker & $X$ & \\
\hline Total & 10 & 9 \\
\hline
\end{tabular}


áreas altos níveis de $\mathrm{P}$ no solo afetam negativamente a ocorrência e a colonização produzida por esses fungos micorrízicos arbusculares.

\section{Agradecimentos}

Ao $\mathrm{CNPq}$, pelas bolsas concedidas respectivamente a C.M.A. Mello (PG) e a L.C. Maia (PQ) e pelo apoio financeiro (INCT-Herbário Virtual da Flora e dos Fungos); à FACEPE (Herbário Virtual de PE); a Camilla Maciel, pela ajuda na coleta; a Everardo Sampaio, pelas críticas e sugestões.

\section{Referências bibliográficas}

Aguilera, L.E.; Gutierrez, J.R. \& Moreno, R.J. 1998. Vesiculo arbuscular mycorrhizae associated with saltbushes Atriplex spp. (Chenopodiaceae) in the Chilean arid zone. Revista Chilena de Historia Natural 71: 291-302.

Araújo, F.S.; Rodal, M.J.N.; Barbosa, M.R.V. \& Martins, F.R. 2005. Repartição da flora lenhosa no domínio da Caatinga. Pp. 15-33. In: Araújo, F.S, Rodal, M.J.N., Barbosa, M.R.V. (Orgs.). Análise das variações da biodiversidade do bioma Caatinga: suporte a estratégias regionais de conservação. Brasília, Ministério do Meio Ambiente.

Beltrano, J. \& Ronco, M.G. 2008. Improved tolerance of wheat plants (Triticum aestivum L.) to drought stress and rewatering by the arbuscular mycorrhizal fungus Glomus claroideum: Effect on growth and cell membrane stability. Brazilian Journal of Plant Physiology 20(1): 29-37.

Borba, M.F. \& Amorim, S.M.C. 2007. Fungos micorrízicos arbusculares em sempre-vivas: subsídio para cultivo e replantio em áreas degradadas. Revista de Biologia e Ciências da Terra 7(2): 20-27.

Carvalho, E.M.S. \& Alencar, P.G. 2000. Ocorrência de fungos micorrízicos vesículo-arbusculares associados a Bauhinia glabra, Jacq. Pesquisa em Foco 8(11): 45-53.

Clark, N.M.; Rillig, M.C. \& Nowak, R.S. 2009. Arbuscular mycorrhizal fungal abundance in the Mojave Desert: Seasonal dynamics and impacts of elevated $\mathrm{CO}_{2}$. Journal of Arid Environments 73: 834-843.

Cochran, W.G. 1950. Estimation of bacterial densities by means of the most probable number. Biometrics 6: 105-116.

Collins, C.D. \& Foster, B.L. 2009. Community-level consequences of mycorrhizae depend on phosphorus availability. Ecology 90(9): 2567-2576.

Covacevich, F.; Marino, M.A. \& Echeverría, H.E. 2006. The phosphorus source determines the arbuscular mycorrhizal potential and the native mycorrhizal colonization of tall fescue and wheatgrass. European Journal of Soil Biology 42: 127-138.

CPRM - Serviço Geológico do Brasil. 2005. Projeto cadastro de fontes de abastecimento por água subterrânea. $11 \mathrm{p}$. Diagnóstico do município de Triunfo, estado de Pernambuco. Mascarenhas, J.C., Beltrão, B.A., Souza Junior, L.C., Galvão, M.J.T.G., Pereira, S.N., Miranda, J.L.F. (Orgs.). Recife, CPRM/PRODEEM.

Douds, D.D. 1994. Relationship between hyphal and arbuscular colonization and sporulation in a mycorrhiza of Paspalum notatum Flugge. New Phytologist 126: 233-237.

Drumond, M.A.; Kiill, L.H.P.; Lima, P.C.F.; Oliveira, M.C.; Oliveira, V.R.; Albuquerque, S.G.; Nascimento, C.E.S. \& Cavalcante, J. 2000. Estratégias para o uso sustentável da biodiverdidade da caatinga. In: Seminário para avaliação e identificação de ações prioritárias para a conservação, utilização sustentável e repartição de benefícios da biodiversidade do bioma Caatinga. Petrolina, Embrapa/Cpatsa, UFPE e Conservation International do Brasil.

Feldmann, F. \& Idczak, E. 1992. Inoculum production of vesicular-arbuscular mycorrhizal fungi for use in tropical nurseries. Pp. 799-817. In: Norris, J.R.; Read, D.J.; Varma, A.K. (Ed.). Techniques for Mycorrhizal Research. Methods in Microbiology. London, Academic Press.
Ferraz, E.M.N.; Rodal, M.J.N. \& Sampaio, E.V.S.B. 2003. Physiognomy and structure of vegetation along an altitudinal gradient in the semi-arid region of northeastern Brazil. Phytocoenologia 33: 71-92.

Francis, R. \& Read, D.J. 1995. Mutualism and antagonism in the mycorrhizal symbiosis, with special reference to impacts on plant community structure. Canadian Journal of Botany 73: 1301-1309.

Gai, J.P.; Christie, P.; Cai, X.B.; Fan, J.Q.; Zhang, J.L.; Feng, G. \& Li, X.L. 2009. Occurrence and distribution of arbuscular mycorrhizal fungal species in three types of grassland community of the Tibetan Plateau. Ecological Research 24: 1345-1350.

Gerdemann, J.W. \& Nicolson, T.H. 1964. Spores of mycorrizal Endogone species extracted from soil by wet sieving and decanting. Transactions of the British Mycological Society 46: 235-244.

Giovanetti, M. \& MOSSE, B. 1980. An evaluation of techniques for measuring vesicular arbuscular mycorrhizal infection in roots. New Phytologist 84: 489-500.

Goto, B.T.; Silva, G.A.; Yano-Melo, A.M. \& Maia, L.C. 2010. Checklist of the arbuscular mycorrhizal fungi (Glomeromycota) in the Brazilian semiarid. Mycotaxon 113: 251-154.

He, X.; Mouratov, S. \& Steinberger, Y. 2002. Temporal and spatial dynamics of vesicular-arbuscular mycorrhizal fungi under the canopy of Zygophyllum dumosum Boiss. in the Negev Desert. Journal of Arid Environments 52: 379-387.

Hijri, I.; Sẏkorová, Z.; Oehl, F.; Ineichen, K.; Mäder, P.; Wiemken, A. \& Redecker, D. 2006. Communities of arbuscular mycorrhizal fungi in arable soils are not necessarily low in diversity. Molecular Ecology 15: 2277-2289.

Jenkins, W.R. 1964. A rapid centrifugal flotation technique for separating nematodes from soil. Plant Disease Reporter 48: 692.

Karanika, E.D.; Voulgaria, O.K.; Mamolosa, A.P.; Alifragisb, D.A. \& Veresogloua, D.S. 2008. Arbuscular mycorrhizal fungi in northern Greece and influence of soil resources on their colonization. Pedobiologia 51: 409-418.

Leal, I.R.; Tabarelli, M. \& Silva, J.M.C. 2003. Ecologia e Conservação da Caatinga. Recife, Editora Universitária da UFPE.

Liu, J.; Maldonado-Mendoza, I.; Lopez-Meyer, M.; Cheung, F.; Town, C.D. \& Harrison, M.J. 2007. Arbuscular mycorrhizal symbiosis is accompanied by local and systemic alterations in gene expression and an increase in disease resistance in the shoots. The Plant Journal 50: 529-544.

Maia, L.C. \& Yano-Melo, A.M. 2005. Role of arbuscular mycorrhizal fungi in saline soil. Pp. 282-302. In: Mehrotra, V.S. (Ed.). Mycorrhiza: Role and Applications. New Delhi, Allied Publishers Pvt.

Mathur, N.; Singh, J.; Bohra, S. \& Vyas, A. 2007. Arbuscular mycorrhizal status of medicinal halophytes in saline areas of India Thar Desert. International Journal of Soil Science 2(2): 119-127.

Mergulhão, A.C.E.S.; Oliveira, J.P.; Burity, H.A. \& Maia, L.C. 2007. Potencial de infectividade de fungos micorrízicos arbusculares em áreas nativas e impactadas por mineração gesseira no semiárido brasileiro. Hoehnea 34(3): 341-348.

Mergulhão, A.C.E.S.; Burity, H.A.; Silva, F.S.B.; Pereira, S.V. \& Maia, L. C. 2010. Glomalin Production and Microbial Activity in Soils Impacted by Gypsum Mining in a Brazilian Semiarid Area. American Journal of Agricultural and Biological Sciences 5: 422-429.

Mohammad, M.J.; Hamad, S.R. \& Malkawi, H.I. 2003. Population of arbuscular mycorrhizal fungi in semi-arid environment of Jordan as influenced by biotic and abiotic factors. Journal of Arid Environment 53: 409-417.

Moreira, J.N.; Lira, M.A.; Santos, M.V.F.; Ferreira, M.A.; Araújo, G.G.L.; Ferreira, R.L.C. \& Silva, G.C. 2006. Caracterização da vegetação de Caatinga e da dieta de novilhos no Sertão de Pernambuco. Pesquisa Agropecuária Brasileira 41: 1643-1651.

Moura, M.S.B; Galvincio, J.D.; Brito, L.T.L.; Souza, L.S.B.; Sá, I.I.S. \& Silva, T.G.F. 2007. Clima e água de chuva no semiárido. http://ainfo. cnptia.embrapa.br/digital/bitstream/CPATSA/36534/1/OPB1515.pdf. (Acesso em 24/06/2012).

Muthukumar, T. \& Udaiyan, K. 2002. Seasonality of vesicular-arbuscular mycorrhizae in sedges in a semi-arid tropical grassland. Acta Oecologica 23: 337-347. 
Oehl, F. \& Sieverding, E. 2004. Pacispora, a new vesicular arbuscular mycorrhizal fungal genus in the Glomeromycetes. Journal of Applied Botany 78: 72-82.

Panwar, J. \& Tarafdar, J.C. 2006. Arbuscular mycorrhizal fungal dynamics under Mitragyna parvifolia (Roxb.) Korth. in Thar Desert. Applied Soil Ecology 34: 200-208.

Phillips, J.M. \& Hayman, D.S. 1970. Improved procedures for clearing roots and staining parasitic and vesicular arbuscular mycorrhizal fungi for rapid assessment of infection. Transactions of the British Mycological Society 55: 158-161.

Schenck, N.C. \& Pérez, Y. 1990. Manual for the identification of VA mycorrhizal fungi. 3th ed. Gainesville, Synergistic Publ.

Schüssler, A.; Schwarzott, H.D. \& Walker, C. 2001. A new fungal phylum, the Glomeromycota: phylogeny and evolution. Mycological Research 105(12): 1413-1421.

Shi, Z.Y.; Zhang, L.Y.; Li, X.L.; Feng, G.; Tian, C.Y. \& Christie, P. 2007. Diversity of arbuscular mycorrhizal fungi associated with desert ephemerals in plant communities of Junggar Basin, northwest China. Applied Soil Ecology 35: 10-20.

Silva, G.A.; Maia, L.C.; Silva, F.S.B. \& Lima, P.C.F. 2001. Potencial de infectividade de fungos micorrízicos arbusculares oriundos de área de caatinga nativa e degradada por mineração, no Estado da Bahia, Brasil. Revista Brasileira de Botânica 24(2): 135-143.

Silva, G.A.; Trufem, S.F.B.; Saggin Júnior, O.J. \& Maia, L.C. 2005. Arbuscular mycorrhizal fungi in a semi-arid copper mining area in Brazil. Mycorrhiza 15: 47-53.
Silva, L.X.; Figueiredo, M.V.B.; Silva, G.A.; Goto, B.T.; Oliveira, J.P. \& Burity, H.A. 2007. Fungos micorrízicos arbusculares em áreas de plantio de leucena e sábia no estado de Pernambuco. Revista Árvore 31: 427-435.

Singh, S.; Pandey, A.; Chaurasia, B. \& Palni, L.M.S. 2008. Diversity of arbuscular mycorrhizal fungi associated with the rhizosphere of tea growing in 'natural' and 'cultivated' ecosites. Biology and Fertility of Soils 44: 491-500.

Smith, S.E. \& Read, D.J. 1997. Mycorrhizal Symbiosis. 2th ed. London, Academic Press.

Souza, R.G.; Maia, L.C.; Sales, M.F. \& Trufem, S.F.B. 2003. Diversidade e potencial de infectividade de fungos micorrízicos arbusculares em área de caatinga, na Região de Xingó, Estado de Alagoas, Brasil. Revista Brasileira de Botânica 26(1): 49-60.

Statsoft. 1994. Statistic for Windows 95. General Convertions and Statistics 1. Oklahoma, Sattsoft Incorporations.

Uhlmann, E.; Görke, C.; Petersen, A. \& Oberwinkler, F. 2006. Arbuscular mycorrhizae from arid parts of Namibia. Journal of Arid Environments 64: 221-237.

Yano-Melo, A.M.; Trufem, S.F.B. \& Maia, L.C. 2003. Arbuscular mycorrhizal fungi in salinized and surrounded areas at the São Francisco Submedium Valley, Brazil. Hoehnea 30(2): 79-87.

Zhao, D. \& Zhao, Z. 2007. Biodiversity of arbuscular mycorrhizal fungi in the hot-dry valley of the Jinsha River, southwest China. Applied Soil Ecology 37: 118-128. 